\title{
Treatment Initiation Patterns, Modifications, and Medication Adherence Among Newly Diagnosed Heart Failure Patients: A Retrospective Claims Database Analysis
}

\author{
Céline Deschaseaux, MSc; Martin McSharry, BSc; Eibhlin Hudson, BA, MA, PhD; \\ Rumjhum Agrawal, MPharm; and Stuart J. Turner, BPharm, MPH
}

\begin{abstract}
BACKGROUND: Heart failure (HF) is a debilitating disease associated with high mortality and frequent hospitalizations. American College of Cardiology Foundation and American Heart Association (ACCF/AHA) guidelines recommend the following drug classes for HF treatment: angiotensin-converting enzyme inhibitor (ACEI), angiotensin receptor II blocker (ARB) for patients intolerant to $A C E I$, beta blocker (BB), and aldosterone antagonist (AA).
\end{abstract}

OBJECTIVE: To examine, in a real-word setting, the treatment initiation pattern among newly diagnosed HF patients in the United States, subsequent treatment modifications, hospitalizations and the impact of hospitalizations on therapy changes, and treatment adherence and persistence.

METHODS: Using medical and pharmacy claims data from the Truven Health MarketScan database, this retrospective cohort study included adult patients with $\geq 2$ medical claims corresponding to an HF diagnosis (ICD-9CM codes 428.x, 402.11, 402.91, 404.01, 404.11, 404.91, 404.03, 404.13, and 404.93) between April 2009 and March 2012. The date of the first claim was defined as the index date. Patients with continuous medical and pharmacy eligibility for a minimum of 12 months pre- and post-index were included in the analysis. Patients with an HF diagnosis in the 12 months before the index date were excluded. Index treatment (within 30 days postindex), subsequent treatment modification (class addition/removal) during the study period, hospitalization, and change in treatment after hospitalization (within 15 days after hospital discharge) were determined. Adherence was evaluated using the proportion of days covered (PDC) method, and persistence was defined as the proportion of patients remaining on index treatment after a defined period of time (12 months).

RESULTS: A total of 235,758 patients meeting the sample selection criteria were included in the analysis and were followed for a median of 28 months after the index date. Approximately $42 \%$ of patients were not prescribed any HF-specific treatment within 30 days post-index. Among those treated, prescriptions for ACEls were filled by $46.42 \%$ of patients, ARBs by $17.07 \%$, BBs by $75.62 \%$, and AAs by $9.83 \%$. Based on HF therapy class, monotherapy was prescribed to $51 \%$ of patients, bi-therapy to $40 \%$, and triple therapy to $9 \%$. More than $80 \%$ of patients experienced treatment modification during the median 28 months of follow-up. A total of $174,563(74.0 \%)$ patients had at least 1 all-cause hospitalization (mean $1.11[S D=0.98]$ ) per year, with a mean length of stay (LOS) of 7.19 [SD =8.69] days. Within 12 months postindex, $85.7 \%$ of these patients experienced an all-cause hospitalization, with 29.6\% having HF-related hospitalization (mean 0.18 [0.36]) and mean LOS of 5.85 [5.45] days. More than $60 \%$ of patients continued on the same therapy after all-cause or HF hospitalization. More patients on multiple therapies remained on the same treatment $(73 \%-89 \%)$ compared with those treated with monotherapy (60\%-73\%) after the first HF hospitalization. Among patients untreated before hospitalization, $9.8 \%$ and $17 \%$ received treatment after all-cause and HF hospitalization, respectively. During the entire study period (median 28 months), $29 \%$ of patients did not have a prescription fill for HF-specific treatments. The median PDC was $>0.65$, and considering a gap of 30 days between ends of supply from 1 medication fill to the subsequent fill, persistence ranged from $41 \%(\mathrm{AA})$ to $52 \%(\mathrm{BB})$.

CONCLUSIONS: Findings of this claims database analysis among 235,758 HF patients suggest that more than one third of newly diagnosed HF patients do not receive HF-specific medication within 30 days following initial diagnosis. Despite ACCF/AHA recommendations of initiating treatment with a combination of $2 \mathrm{HF}$ drug classes, only $40 \%$ of patients had a prescription fill for bi-therapy. Hospitalization did not have a major impact on HF therapy prescribing patterns. To our knowledge, this is the first study to establish the impact of hospitalization on HF-specific treatment among newly diagnosed patients. Adherence and persistence were moderate across all HF therapies of interest. This analysis reveals the need for further research to better understand the reasons for the demonstrated delay in $\mathrm{HF}$ treatment initiation and limited use of guideline-directed medical therapy after initial diagnosis.

J Manag Care Spec Pharm. 2016;22(5):561-71

Copyright $\odot 2016$, Academy of Managed Care Pharmacy. All rights reserved.

\section{What is already known about this subject}

Heart failure (HF) is a global public health problem affecting an estimated of 5.7 million patients in the United States. Approximately $50 \%$ of patients who develop HF die within 5 years of diagnosis, and in the United States, more than 1 million hospitalizations annually are attributed to HF.

American College of Cardiology Foundation/American Heart Association guidelines recommend a combination of angiotensinconverting enzyme inhibitors (ACEI)/angiotensin receptor II blockers (ARB) and beta blockers (BB) for the management of HF. Aldosterone antagonists are recommended as an add-on therapy for all patients with persisting symptoms despite treatment with ACEIs/ARBs and BBs.

Previous studies have shown that these therapies are often under prescribed.

\section{What this study adds}

This large, population-based study reflects the real-world clinical practice and treatment adherence of over $200,000 \mathrm{HF}$ patients in the United States. 


\section{What this study adds (continued)}

This study found that a substantial proportion of patients (42\%) did not receive HF-specific medication within 30 days after diagnosis, and $29 \%$ of patients did not have a prescription fill for HF-specific treatments during the entire study period (median 28 months). This finding highlights the need for increased usage of guideline-directed medical therapy.

Data on the impact of hospitalization on treatment showed that more than $60 \%$ of patients continued on the same therapy after all-cause or HF-related hospitalization. Among patients who were untreated before an all-cause hospitalization, $9.8 \%$ received treatment after the hospitalization, and $17 \%$ received treatment after an HF-related hospitalization.

$\mathrm{I}$ $\mathrm{n}$ the United States, heart failure (HF) affects approximately 5.7 million adults, with 870,000 new cases per year. ${ }^{1}$ The prevalence of HF is projected to approximately double by $2030 .^{2}$ HF is associated with increased morbidity and mortality and a high economic burden primarily driven by hospitalizations. ${ }^{3,4}$

The American College of Cardiology Foundation/American Heart Association (ACCF/AHA) guidelines recommend a combination of angiotensin-converting enzyme inhibitors (ACEIs), or angiotensin receptor blockers (ARBs) if intolerant to ACEIs, and beta blockers (BBs) as first-line treatment for HF with reduced ejection fraction (HFrEF) patients. Since there is no evidence-based therapy for the treatment of HF patients with preserved ejection fraction (HFpEF), guidelines advise management of symptoms and comorbidities. Specific medications in these treatment classes are the cornerstone of HF treatment, since they have been proven to improve symptoms, increase survival, and decrease hospitalizations. ${ }^{3}$ In cases where patients require more management, aldosterone antagonists (AAs) are recommended in cases where patients are symptomatic despite treatment with ACEIs and BBs.

Unfortunately, although multiple studies have shown that there is an improvement in survival post-HF diagnosis, the 5 -year mortality rate remains considerably high at 50\%., $\mathrm{HF}$ is the first cause of hospitalization ahead of cancer and respiratory diseases in patients aged $>65$ years and accounts for 3\% of all hospital admissions in the United States, with nearly 1 million hospitalizations annually. ${ }^{6-8}$ There is inconsistent evidence for trends in hospitalizations over time; recent AHA statistics report no change in prevalence of HF hospitalizations from 2000 to 2010. ${ }^{1}$ However, past studies report a decline in primary HF hospitalizations during the periods from 1998 to 2007 and 2001 to $2009 .{ }^{9,10}$

Despite published recommendations from the ACCF/AHA, various studies have shown that HF patients are suboptimally managed, and treatment guidelines are often not followed in clinical practice. ${ }^{11-14}$ Furthermore, multiple comorbidities add complexity to the effective disease management of HF patients. Cumulatively, these result in adherence and persistence challenges, which may have negative implications for HF care and treatment outcomes.

The objective of this study was to examine treatment initiation patterns and subsequent treatment modifications, hospitalizations and the impact of hospitalizations on therapy changes, and medication adherence and persistence among newly diagnosed HF patients in a U.S. real-world setting.

\section{Methods}

\section{Study Design and Data}

A retrospective observational study was conducted using administrative claims data from the Truven Health MarketScan Commercial Claims and Encounters (CCAE) database and the Medicare Supplemental and Coordination of Benefits database.

The CCAE database contains employer-sponsored private health insurance records encompassing employees and their spouses and dependents. The Medicare Supplemental and Coordination of Benefits database contains the data of retirees with employer-sponsored Medicare supplemental insurance. ${ }^{15}$ MarketScan databases are in compliance with the Health Information Portability and Accountability Act of 1996 (HIPAA). The MarketScan claims data used in the current analysis covered the period from April 1, 2008, to September 30,2013 , since this was the most recent data available at the time of this analysis.

\section{Sample Selection}

Adult patients ( $>18$ years of age) with at least 2 medical claims within 12 months on different dates corresponding to a HF diagnosis during the period from April 1, 2009, to March 31, 2012, were identified. The date of the first of these claims was defined as the index date (Figure 1). HF diagnosis was based on the International Classification of Diseases, Ninth Revision, Clinical Modification (ICD-9-CM) codes 428.x, 402.11, 402.91, 404.01, 404.11, 404.91, 404.03, 404.13, and 404.93. Patients were required to have continuous enrollment in the database for 1 year before the diagnosis of congestive HF and at least 1 year of follow-up data after the index date. To ensure that prevalent patients were not included, patients with HF diagnosis in the 12 months pre-index period were excluded. Patients with multiple comorbidities were not excluded.

\section{Study Variables}

Patient characteristics assessed at the index date included age, gender, region, health insurance plan type, and employment status. Patient history was identified using ICD-9-CM codes, and the Charlson Comorbidity Index (CCI) was calculated for the 12-month pre-index period (baseline). 


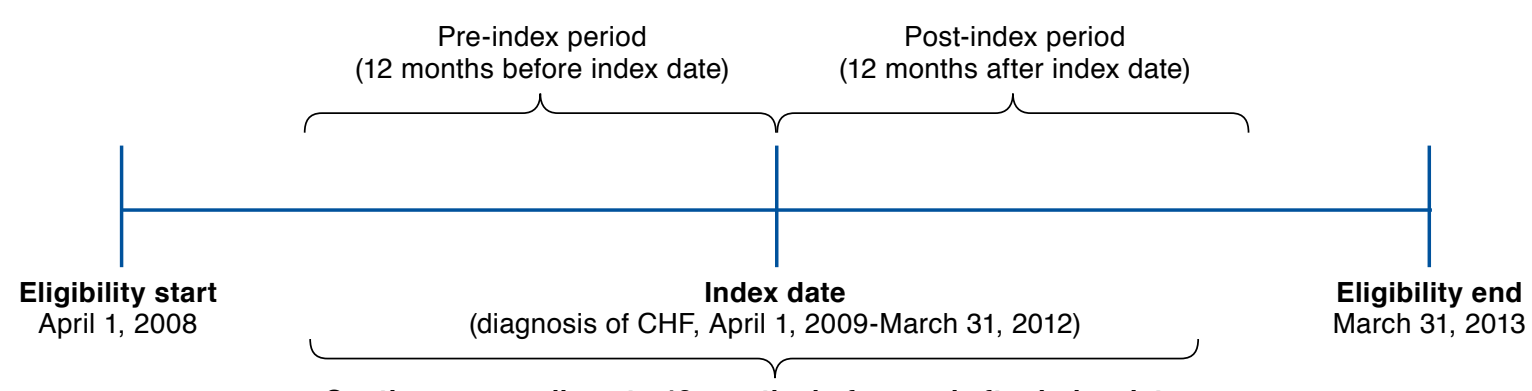

Continuous enrollment $\geq 12$ months before and after index date
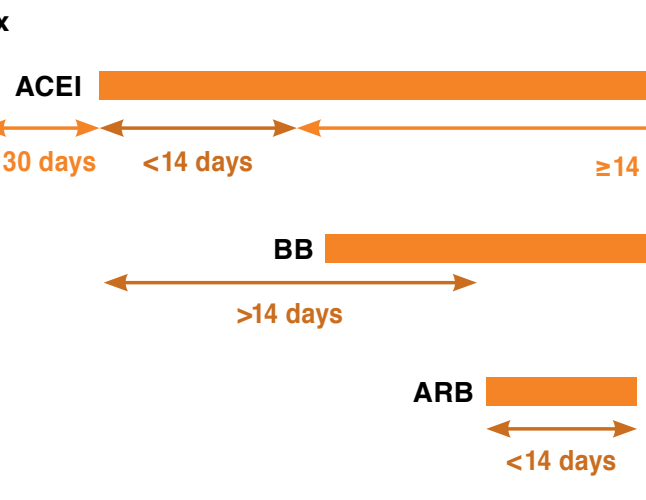

\section{Index+30}

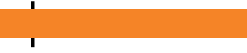

\section{$\rightarrow$}
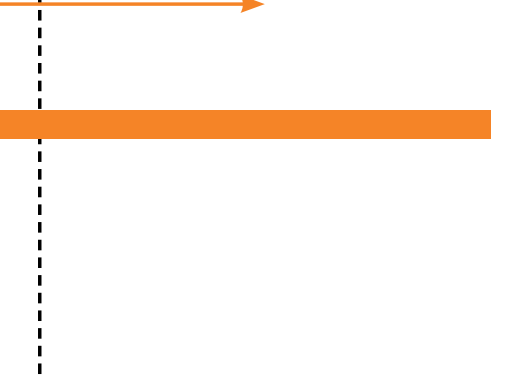

ACEI+BB

$A C E I=$ angiotensin-converting enzyme inhibitor; $A R B=$ angiotensin receptor II blocker; $B B=$ beta blocker

Drug treatment at index and end of follow-up was identified for the following treatment classes: ACEI, ARB, AA, BB, and diuretics (any). Diuretics were not included in combination treatments, since they are not disease-modifying drugs and are given mainly for management of symptoms. Due to the unavailability of dosage information in the database, treatment modifications were assessed only at the class level. Patients were grouped based on the number of treatment classes prescribed: monotherapy (1 treatment class), bi-therapy (a combination of 2 treatment classes), triple therapy (a combination of 3 treatment classes), and quadruple therapy (a combination of 4 treatment classes). The criteria for qualifying index treatments were as follows (Figure 2):

- The earliest drug class used was set as index therapy.

- Index therapy included drugs taken for at least 14 days within 30 days of index date.

- Other drug classes started within 14 days of index therapy, and continued together with index therapy for at least 14 days, were added to the index therapy and considered as combination treatment.

Device treatment such as implantable cardioverter defibrillator (ICD)/cardiac resynchronization therapy (CRT) was also identified. First treatment modification (defined as a change in treatment class or a change of combination [a new treatment class added or removed]) and time from index until the first treatment modification were evaluated.

All-cause and primary HF-related hospitalizations were estimated after the index date, with index date inclusive, and treatment transition after hospitalization (within a 15-day period of hospital discharge) was determined. Hospital treatment changes were only assessed if the data were available before and after hospitalization.

Adherence was evaluated using the proportion of days covered (PDC) method. ${ }^{16}$ Persistence was defined as the proportion of patients remaining on index treatment after a defined period 


\section{FIGURE 3 Patient Selection Process}

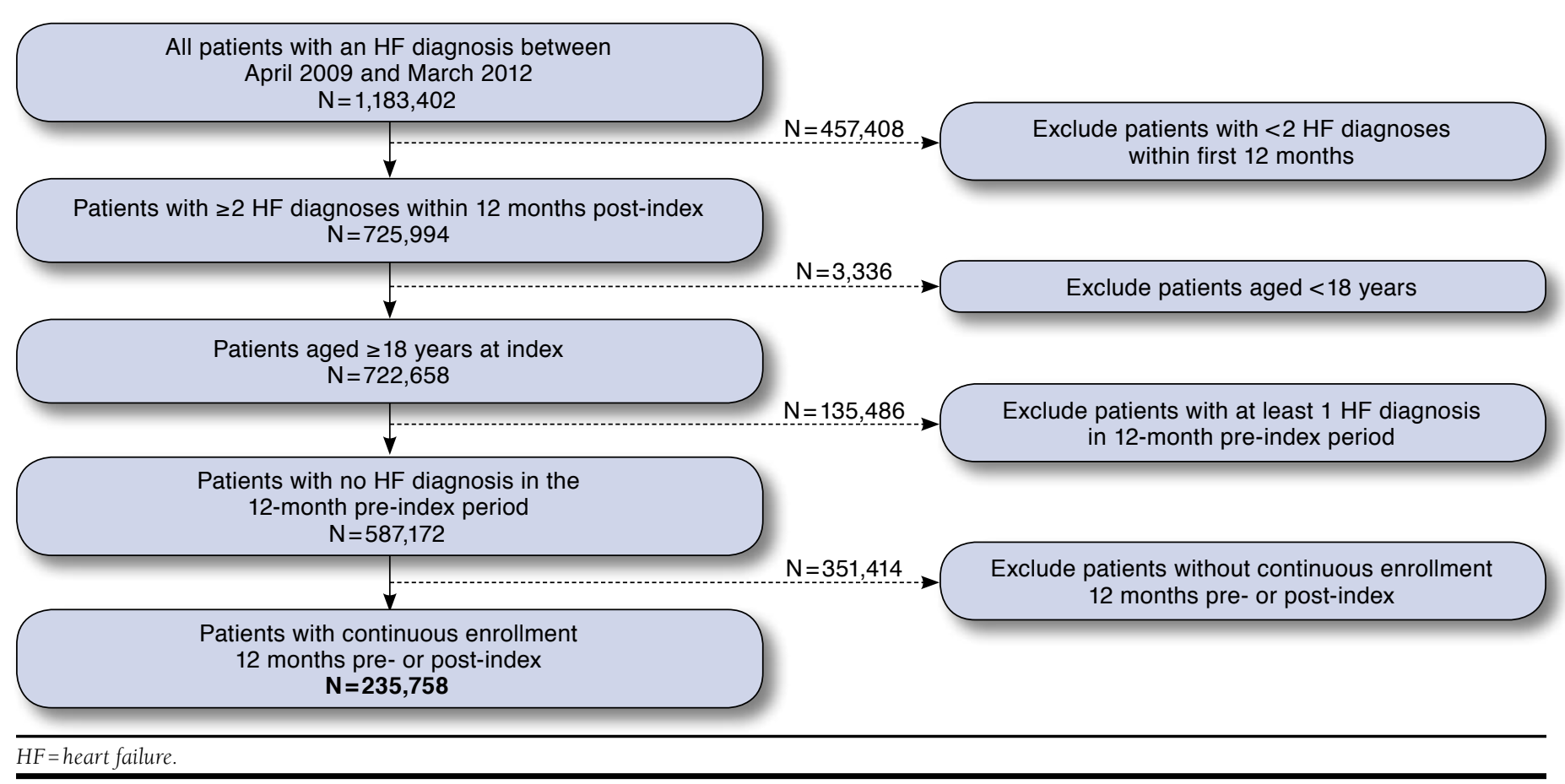

of time (12 months). A treatment gap period of a maximum of 30 days was regarded as acceptable between the end of supply from 1 medication fill to the subsequent fill. ${ }^{16}$

\section{Analysis}

Data were summarized using descriptive analyses. For categorical variables, counts and percentages were provided for each treatment class.

\section{Results}

After inclusion and exclusion criteria were met, 235,758 newly diagnosed HF patients comprised the study cohort (Figure 3). Patients were followed up for a median of 28 months (range $=12.2-54.8$ months) after the index date.

\section{Baseline Characteristics}

A summary of patient demographics and clinical characteristics is presented in Table 1. Mean age of the study cohort was 70.9 years (standard deviation $[S D]=14.1$ ) with $46.5 \%$ of the patients aged $\geq 75$ years. There was a relatively even gender split with male patients comprising $51.6 \%$ of the cohort. Similar percentages of patients were from the North Central region $(31.3 \%)$ and South region (30.2\%).

The majority of patients in the study cohort had a history of essential hypertension (69.9\%), followed by type 2 diabetes
(37.3\%) and atrial fibrillation (28.3\%). An equal percentage of patients had a CCI of 1 and $4+(24.7 \%$ and $24.8 \%$, respectively).

\section{Treatment Initiation Pattern}

Overall, 41.8\% ( $n=98,630)$ of patients did not have any single prescription fill for HF-specific treatments of interest (ACEIs, ARBs, BBs, and AAs) within 30 days of their first diagnosis. Even after taking diuretics into consideration, there were 35.5\% $(n=83,710)$ of patients who did not have a treatment prescribed within 30 days of initial diagnosis. Among patients treated with HF-specific treatments $(n=137,128)$, prescriptions for ACEIs were filled by $46.42 \%$, ARBs by $17.07 \%$, BBs by $75.62 \%$, and AAs by $9.83 \%$. Overall, $51.3 \%$ of patients had prescription fills for monotherapy, and $40 \%$ for bi-therapy (Figure 4). Among the treated cohort, BB prescription fills were the most common monotherapy fills (29.8\%), followed by ACEI (13.6\%), ARB (6.3\%), ACEI/ARB (19.9\%), and AA (1.6\%) fills. Prescriptions for bi-therapy of ACEI + BB were filled by $28.2 \%$ of patients, $\mathrm{ARB}+\mathrm{BB}$ by $9.4 \%$, and $\mathrm{BB}+\mathrm{AA}$ by $2.4 \%$. Prescriptions for the triple therapy combination of $\mathrm{ACEI}+\mathrm{BB}+\mathrm{AA}$ were filled by $4.4 \%$ of patients and $A R B+B B+A A$ by $1.2 \%$. In total, $66.1 \%$ of the treated patients had at least 1 prescription fill for ACEIs/ ARBs, $76.8 \%$ for BBs, and $11.1 \%$ for AAs during the first 30 days after diagnosis. 


\begin{tabular}{|c|c|c|}
\hline \multirow{2}{*}{$\begin{array}{l}\text { Characteristic } \\
\text { Age, years, mean (SD) }\end{array}$} & \multicolumn{2}{|c|}{$\mathrm{N}=235,758$} \\
\hline & 70.9 & $(14.1)$ \\
\hline $18-64, \mathrm{n}(\%)$ & 81,060 & $(34.4)$ \\
\hline $65-74, \mathrm{n}(\%)$ & 45,038 & (19.1) \\
\hline $75+, \mathrm{n}(\%)$ & 109,660 & $(46.5)$ \\
\hline Male, n (\%) & 121,693 & $(51.6)$ \\
\hline \multicolumn{3}{|l|}{ Region, n (\%) } \\
\hline Northeast & 43,618 & $(18.5)$ \\
\hline North Central & 73,894 & (31.3) \\
\hline South & 71,115 & $(30.2)$ \\
\hline West & 41,669 & $(17.7)$ \\
\hline Unknown & 5,462 & $(2.3)$ \\
\hline \multicolumn{3}{|l|}{ Plan type, n (\%) } \\
\hline $\mathrm{PPO}$ & 93,577 & $(39.7)$ \\
\hline Comprehensive & 87,901 & $(37.3)$ \\
\hline $\mathrm{HMO}$ & 29,648 & $(12.6)$ \\
\hline POS & 11,377 & $(4.8)$ \\
\hline CDHP & 3,606 & $(1.5)$ \\
\hline EPO & 1,902 & $(0.8)$ \\
\hline HDHP & 519 & $(0.2)$ \\
\hline POS with capitation & 519 & $(0.2)$ \\
\hline Missing/unknown & 6,190 & $(2.6)$ \\
\hline \multicolumn{3}{|l|}{ Employment status, n (\%) } \\
\hline Medicare eligible retiree & 78,705 & $(33.4)$ \\
\hline Active full time & 24,109 & $(10.2)$ \\
\hline Surviving spouse/dependent & 14,262 & $(6.0)$ \\
\hline Retiree (status unknown) & 14,004 & (5.9) \\
\hline Early retiree & 9,856 & $(4.2)$ \\
\hline Other/unknown & 93,348 & $(39.6)$ \\
\hline \multicolumn{3}{|l|}{ Medical history, \% } \\
\hline Hypertension & \multicolumn{2}{|c|}{69.9} \\
\hline Type 2 diabetes & \multicolumn{2}{|c|}{37.3} \\
\hline Atrial fibrillation & \multicolumn{2}{|c|}{28.3} \\
\hline COPD & \multicolumn{2}{|c|}{23.9} \\
\hline Renal impairment/failure & \multicolumn{2}{|c|}{22.9} \\
\hline Stroke & \multicolumn{2}{|c|}{20.1} \\
\hline Myocardial infarction & \multicolumn{2}{|c|}{13.3} \\
\hline \multicolumn{3}{|c|}{ Charlson Comorbidity Index, n (\%) } \\
\hline 0 & 39,335 & $(16.7)$ \\
\hline 1 & 58,229 & $(24.7)$ \\
\hline 2 & 46,027 & $(19.5)$ \\
\hline 3 & 33,773 & $(14.3)$ \\
\hline $4+$ & 58,394 & $(24.8)$ \\
\hline
\end{tabular}

$\mathrm{CDHP}=$ consumer -driven health plan; $\mathrm{COPD}=$ chronic obstructive pulmonary disease; $E P O=$ exclusive provider organization; $H D H P=$ high-deductible health plan; $\mathrm{HF}=$ heart failure; $\mathrm{HMO}=$ health maintenance organization; $\mathrm{PPO}=$ preferred provider organization; $P O S=$ point of service; $S D=$ standard deviation.

In addition to the specific HF treatment classes, $74.8 \%$ of patients had prescription fills for diuretics. Prescription fills for fixed dose combinations of ACEIs (3.0\%) or ARBs (4.3\%) were limited. Usage of ICD/CRT was also limited, with only $2 \%$ of patients having an ICD/CRT-related claim.

\section{Treatment Modifications}

Among patients treated at index, treatment modification (initiation, discontinuation, or modification of treatment) at the end of the post-index follow-up period (median 28 months) was reported in more than $80 \%$. The median time to first treatment modification was 3.0 months (range $=2.1$ months for AAs to 3.3 months for $\mathrm{BBs}$ ) for patients receiving monotherapy; 3.6 months (range $=2.6$ months for $\mathrm{BB}+\mathrm{AA}$ to 3.8 months for $\mathrm{ARB}+\mathrm{BB}$ ) for bi-therapy; 3.2 months for triple therapy; and 1.4 months for patients receiving quadruple therapy of $\mathrm{ACEI}+\mathrm{ARB}+\mathrm{BB}+\mathrm{AA}$.

The most frequent treatment modifications observed at the end of the follow-up period are presented in Figure 5 . De-escalation of therapy during follow-up was reported for the majority of patients, with those on bi-therapy and triple therapy transitioning to monotherapy and bi-therapy, respectively. The most common transitions for patients on monotherapy were as follows: ACEI to ACEI + BB (27.1\%), ARB to ARB + BB (25.9\%), $\mathrm{AA}$ to $\mathrm{AA}+\mathrm{BB}(17.8 \%)$, and $\mathrm{BB}$ to $\mathrm{BB}+\mathrm{ACEI}(17.5 \%)$. The cumulative proportion of patients with an ICD/CRT-related claim in the post-index period was $3.9 \%$ at 1 year, $4.7 \%$ at 2 years, $5.0 \%$ at 3 years, $5.2 \%$ at 4 years, and $5.2 \%$ at 5 years.

Among initially untreated patients $(n=98,630), 31.4 \%$ of patients received treatment after a median of 4.7 months. Specifically, these patients received BB monotherapy (44.6\%), ACEI monotherapy (21.8\%), or ARB monotherapy (9.8\%). Of the total study cohort, $29 \%$ of patients $(n=67,672)$ did not have a prescription fill for HF-specific treatments (ACEI, ARB, BB, or AA) during the entire study period (median 28 months). After including diuretics, there were still $24.9 \%$ of patients $(n=58,812)$ without a prescription fill during the entire study period.

\section{Hospitalizations and Change in Treatment}

A total of $174,563(74.0 \%)$ patients had at least 1 all-cause hospitalization with a mean of $1.11(\mathrm{SD}=0.98)$ hospitalizations per year and length of stay (LOS) of 7.19 (8.69) days. Of these 174,563 patients, $149,593(85.7 \%)$ patients were hospitalized within the first 12 months. Similarly, 51,635 (21.9\%) patients had HF-related hospitalizations, with a mean of $0.18(0.36)$ hospitalizations per year and mean LOS 5.85 (5.45) days.

Overall, $80.5 \%$ of patients continued on the same treatment regimen following the first all-cause hospitalization (within 15 days). Transitioning to ACEI +BB bi-therapy was reported in $13 \%$ and $6 \%$ patients initially treated with ACEI or BB monotherapy, respectively (Figure 6). Among patients on bi-therapy with BBs, transitioning to BB monotherapy was the most frequent treatment modification. Of the untreated patients, 9.8\% received treatment after all-cause hospitalization.

Following the first HF-related hospitalization, no change in treatment was observed for $74.7 \%$ of the patients. More patients on bi- and triple therapy remained on the same 
Treatment Initiation Patterns, Modifications, and Medication Adherence Among Newly Diagnosed Heart Failure Patients: A Retrospective Claims Database Analysis

\section{FIGURE 4 Treatment Distribution Among Incident HF Patients at Index (Within 30 Days)}
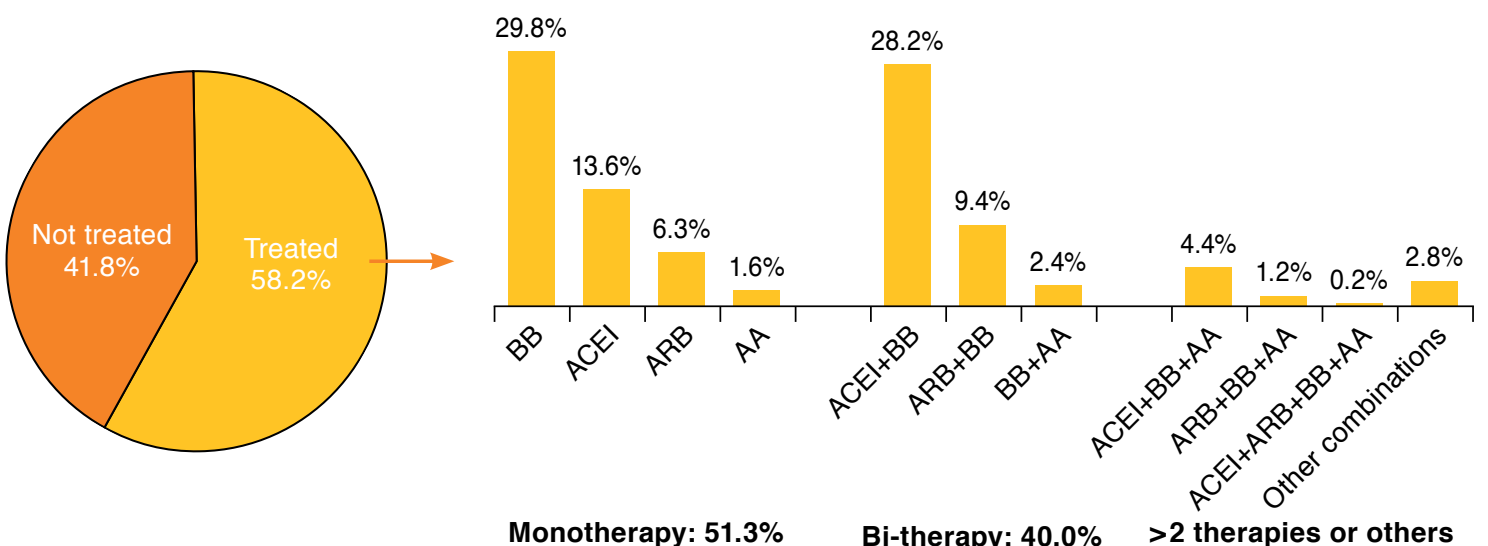

$A A=$ aldosterone antagonist; $A C E I=$ angiotensin-converting enzyme inhibitor; $A R B=$ angiotensin receptor II blocker; $B B=$ beta blocker; $H F=$ heart failure.

FIGURE 5 Top 2 Treatment Modifications at End of Follow-up Period

Initial Therapies

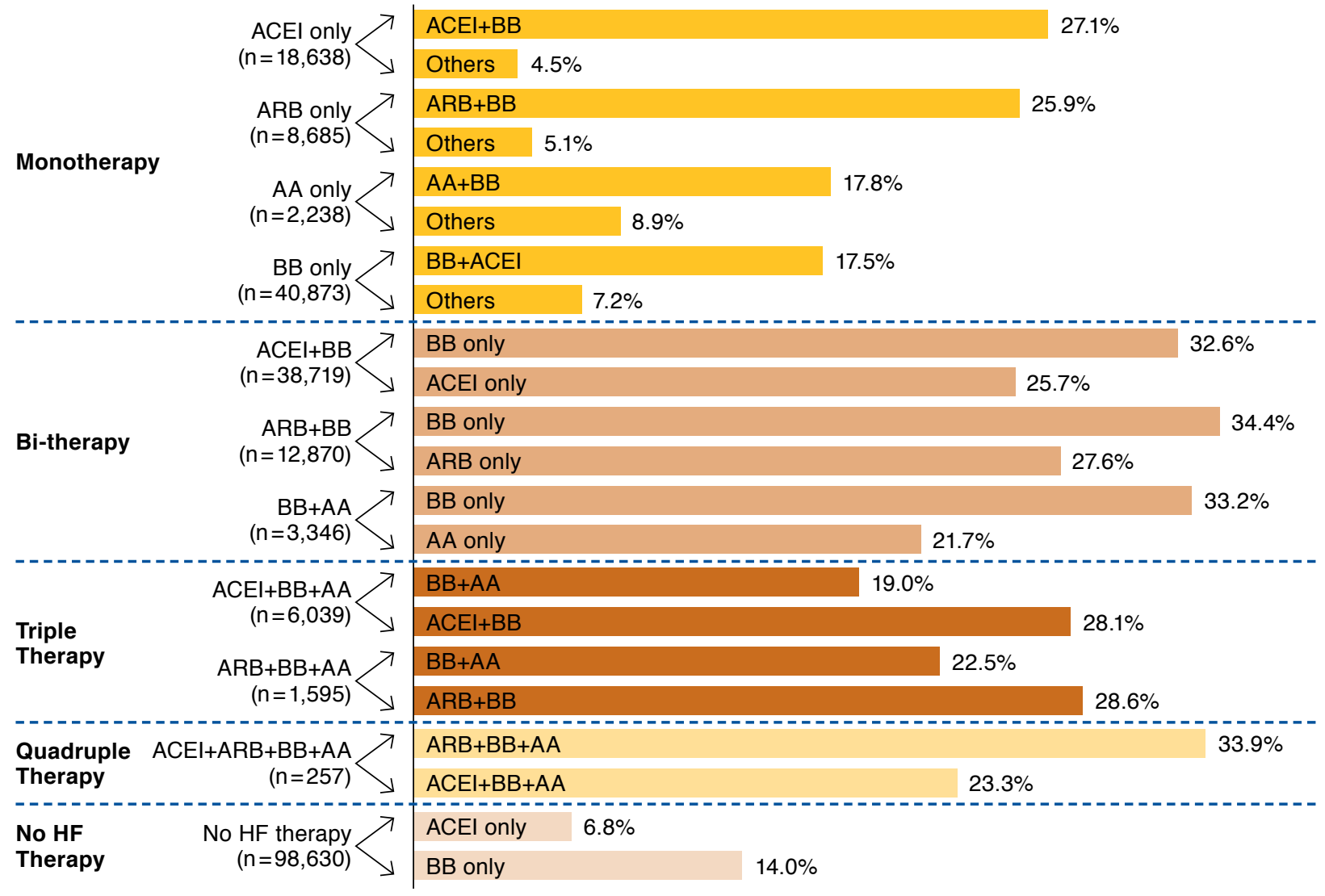

Note: Horizontal bars represent the proportion of patients that underwent treatment modifications at the end of follow-up (the top 2).

$A A=$ aldosterone antagonist; $A C E I=$ angiotensin-converting enzyme inhibitor; $A R B=$ angiotensin receptor II blocker; $B B=$ beta blocker; $H F=$ heart failure. 
Treatment Initiation Patterns, Modifications, and Medication Adherence Among Newly Diagnosed Heart Failure Patients: A Retrospective Claims Database Analysis

\section{FIGURE 6 Percentage of Patients Switching to Another Combination After First All-Cause Hospitalization} (the 2 Most Frequent Changes)

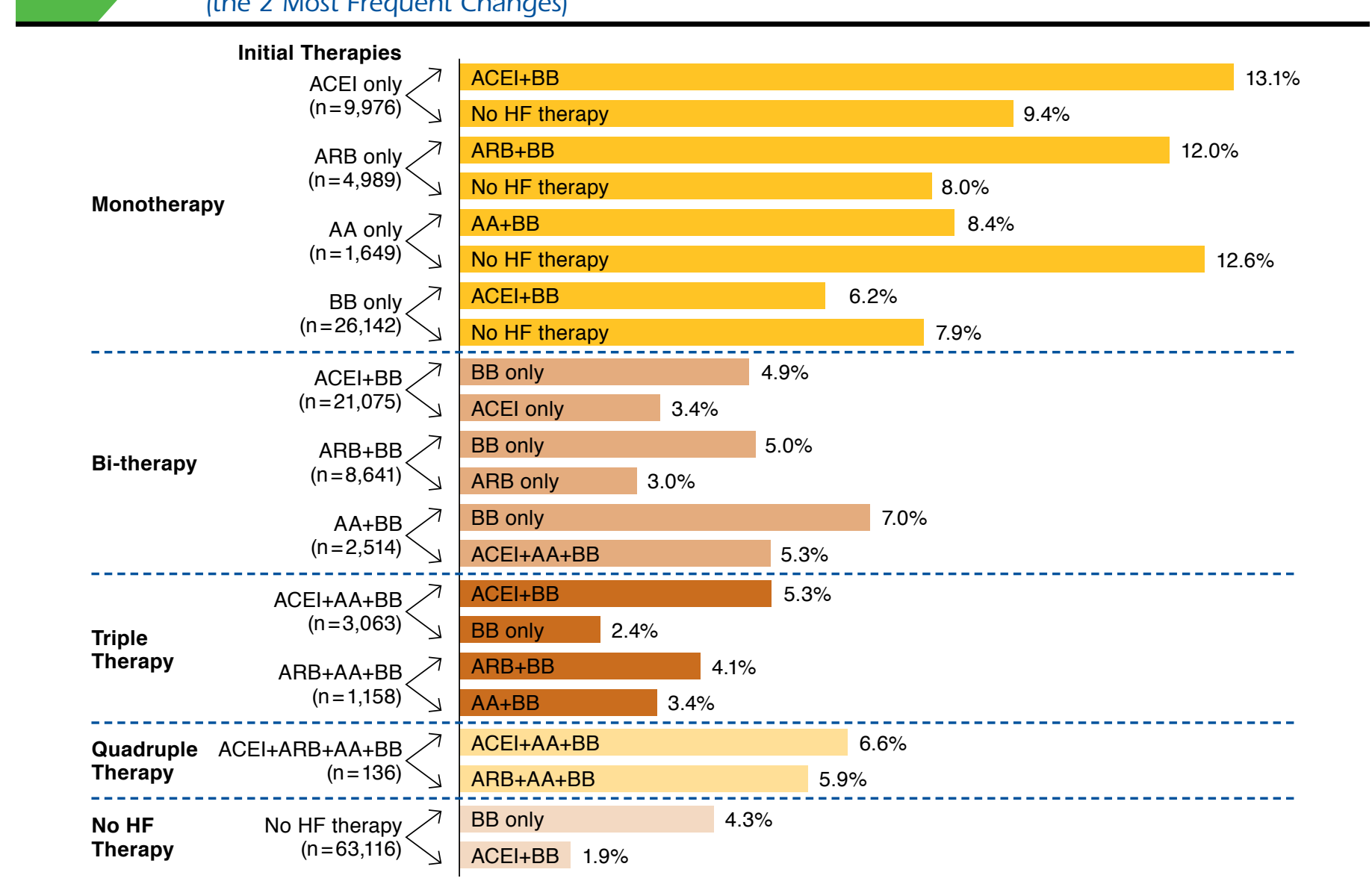

Note: Horizontal bars represent the number of patients that switched treatments (the top 2) after first all-cause hospitalization.

$A A=$ aldosterone antagonist; $A C E I=$ angiotensin-converting enzyme inhibitor; $A R B=$ angiotensin receptor II blocker; $B B=$ beta blocker; $H F=$ heart failure.

treatment (73\%-89\%) compared with those patients treated with monotherapy (60\%-73\%; Figure 7). Among patients on ACEI or BB monotherapy, $21 \%$ and $10 \%$ transitioned to ACEI + BB combination, respectively. Of patients on bi-therapy with BBs, the 2 most frequent transitions were switching to triple therapy, followed by switching to BB monotherapy. Among patients who were untreated before HF-related hospitalization, $17 \%$ received treatment after HF-related hospitalization.

\section{Adherence and Persistence}

Median PDC across HF treatment classes was above 0.65 (Table 2). For all 4 main treatment classes (ACEI, ARB, BB and AA), persistence ranged from $41 \%$ (AA) to $52 \%$ (BB). The findings did not change substantially when the gap period was increased to 45 days (range $=46 \%-60 \%$ ) or 60 days (range $=49 \%-65 \%$; Table 2).

\section{Discussion}

This real-world study presents findings on treatment initiation patterns, treatment modifications, the impact of hospitalization on treatment, and medication adherence for newly diagnosed HF patients based on administrative claims. The analysis highlights that a substantial proportion of newly diagnosed HF patients (41.8\%) did not have a claim for guideline-recommended HF treatment (ACEI, ARB, BB or AA) within 30 days after diagnosis. Furthermore, 29\% of patients did not receive HF therapy by the end of the post-index follow-up, thereby reinforcing findings from previous studies that HF treatments are likely underutilized in the real world. ${ }^{11,12}$ Treatment modification was frequent and was reported in more than $80 \%$ of the patients. The median time to first treatment modification ranged from 1.4 months to 3.8 months. In addition, hospitalization was not associated with a change in treatment within 15 days of discharge in $>70 \%$ of patients. 
Treatment Initiation Patterns, Modifications, and Medication Adherence Among Newly Diagnosed Heart Failure Patients: A Retrospective Claims Database Analysis

FIGURE 7 Percentage of Patients Switching To Another Combination After First HF Hospitalization (the 2 Most Frequent Changes)

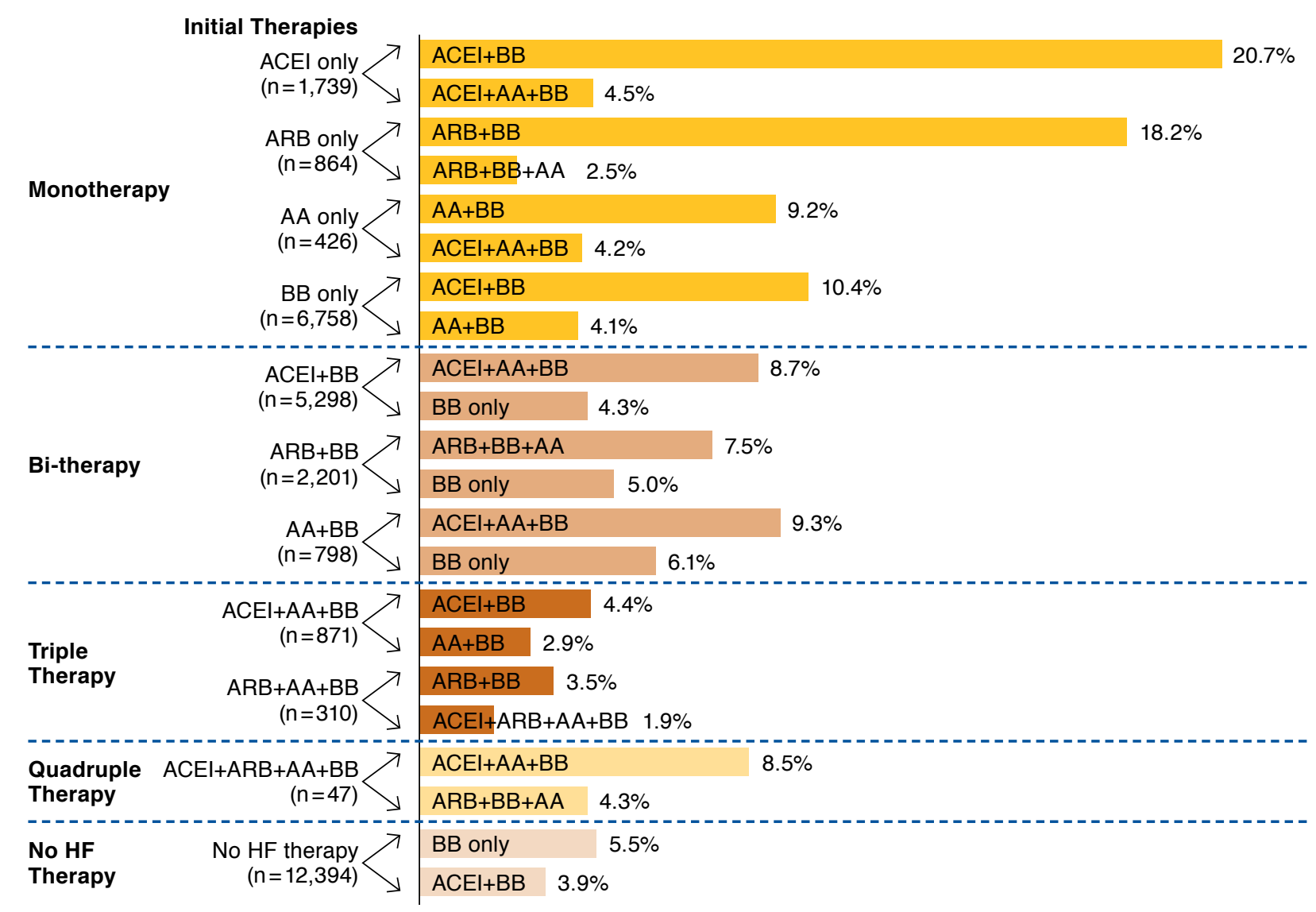

Note: Horizontal bars represent the number of patients that switched treatments (the top 2) after first HF-related hospitalization.

$A A=$ aldosterone antagonist; $A C E I=$ angiotensin-converting enzyme inhibitor; $A R B=$ angiotensin receptor II blocker; $B B=$ beta blocker; $H F=$ heart failure.

As previously noted, $41.8 \%$ of the patients did not have a prescription fill for HF-specific treatment (ACEI, ARB, BB, or AA) within 30 days after diagnosis. This might be partly explained by the inability of the administrative claims database to distinguish New York Heart Association (NYHA) class or to differentiate between HFpEF versus HFrEF patients. Hence, the sample is inclusive of NYHA class I patients, as well as those with HFpEF, for whom there is no approved therapy. Of these untreated patients, 31.4\% received treatment after a median of approximately 4 months, suggesting a delay in HF treatment initiation. Overall, $29 \%$ of patients in the study cohort remained untreated at the end of follow-up. An earlier retrospective analysis based on a longitudinal, patient-level database studied patients from January 1, 1997, to December 31,1999 . This study also reported that $32.3 \%$ of patients did not receive any of the HF therapies (ACEI, BB, loop diuretics, or digoxin). ${ }^{14}$
Despite guideline recommendations to initiate treatment with bi-therapy of ACEIs (ARBs in patients with intolerance to ACEIs) and BBs, more patients had a prescription fill for monotherapy (51.4\%) than bi-therapy (40\%). However, since the analysis included incident patients, the percentage of patients on bi-therapy seems acceptable. Given the age of patients at baseline (mean age 70.9 years), the increased prevalence of contraindications and comorbidities in elderly patients may explain the lower rate of ACEI/ARB vs. BB prescription fills. Other studies evaluating prescription dispensing patterns in a community setting or using administrative claims databases reported similar findings. ${ }^{17-19}$ The study using data from the National Ambulatory Medical Care Survey reported usage of ACEIs/ARBs by $32 \%$ of patients and BBs by $38 \%$, which is lower than the values reported in our study. ${ }^{20}$ Among the registry-based studies, the findings from the "Get With the Guidelines-Heart Failure (GWTG-HF) Registry" were 
Treatment Initiation Patterns, Modifications, and Medication Adherence Among Newly Diagnosed Heart Failure Patients: A Retrospective Claims Database Analysis

TABLE 2 Adherence and Persistence to HF Medications

\begin{tabular}{|c|c|c|c|c|}
\hline & ACEI & ARB & AA & BB \\
\hline PDC, median (range) & $0.759(0.01-1.00)$ & $0.825(0.01-1.00)$ & $0.654(0.01-1.00)$ & $0.886(0.01-1.00)$ \\
\hline Persistence rate, 30-day gap, \% & 44.7 & 47.4 & 41.0 & 52.2 \\
\hline Persistence rate, 45-day gap, \% & 50.5 & 53.7 & 45.8 & 59.8 \\
\hline Persistence rate, 60 -day gap, $\%$ & 54.3 & 57.7 & 49.0 & 64.8 \\
\hline
\end{tabular}

consistent with our study, ${ }^{21}$ whereas data from the IMPROVE-HF registry were relatively higher. ${ }^{22}$ The differences seen in our study compared with the observational registries mentioned may reflect the clinical quality improvement objective present in the scope of most registries. Hence, the practice patterns observed in such registries may be reflective of a more intensively managed population. Our study includes data from privately insured individuals across a variety of practice settings, including nonteaching and nonurban outpatient clinics, whereby many factors including patient adherence, socioeconomic influences, and physician awareness of guidelines may impact treatment patterns.

The baseline usage of ICD/CRT was $2.0 \%$, and there was no substantial increase during the study follow-up $(5.2 \%$ at 5 years post-index). This ICD/CRT rate is lower compared with other studies. The study based on the GWTG-HF registry reported a usage of $19.4 \%$ for ICD and $14.8 \%$ for CRT. ${ }^{21}$ This difference can be explained by the fact that our study included a relatively healthier, newly diagnosed population.

Over the study period, treatment modifications were reported in more than $80 \%$ of patients, with the majority reporting de-escalation in therapy. This is an interesting finding, and although it can be argued that this is because of the inclusion of patients with mild HF symptoms, we cannot rule out the possibility of other potential reasons, such as lack of adherence by patients, treatment tolerability, frequent comorbidities, depression, and various economic and administrative reasons. It should be noted that since information on dosage was unavailable in the database, treatment modifications are assessed only at the class level.

In the United States, 3\% of all hospital admissions have HF as a primary diagnosis. ${ }^{6}$ Hospitalization is the key cost driver and accounts for $60 \%-70 \%$ of the direct medical costs of HF treatment. ${ }^{2}$ The main determinant of HF hospitalization cost is LOS, with a recent review reporting a median LOS less than 5 days for an acute HF episode in North America. ${ }^{23}$ Furthermore, each additional day of initial hospital stay is associated with a significant risk of rehospitalization and mortality within 30 days and 1 year. ${ }^{24}$ Therefore, treatments aiming at reducing HF-related hospitalization will help in limiting overall HF-related health care costs. Compared with LOS data from the OPTIMIZE-HF registry (mean $=5.7, \mathrm{SD}=5.9$ days), ${ }^{25}$ the mean LOS of $7.19(\mathrm{SD}=8.69)$ days for all-cause hospitalizations was higher in the data presented here. However, the mean LOS of 5.85 ( $\mathrm{SD}=5.45)$ days for HF-related hospitalizations was similar to those reported in the published literature (5.27 to 6.3 days). $)^{9,26}$

In the current study, hospitalization did not appear to have a major impact on the prescribing pattern, with $80 \%$ and $74 \%$ of patients continuing on the same treatment after all-cause and HF-related hospitalizations, respectively. It should be noted that patients with index hospitalizations will have more changes in treatment, since these are newly diagnosed patients. Contrary to this, patients with post-index hospitalizations would be expected to have comparatively fewer changes in treatment. A previous study based on the GWTG-HF registry also reports that the majority of patients continued the guideline-recommended medical therapy from hospital admission to discharge..$^{21}$ Of the patients with no HF therapy pre-hospitalization, only $9.8 \%$ and $16.9 \%$ started treatment after all-cause and HF-related hospitalizations, respectively. These rates may be explained by either the short time window of 15 days after hospitalization used to identify treatment initiation, or the physician might see the patient within 15 days; however, the fill date might be later than the 15-day window, or the physician might prescribe an increase/decrease in the dose of certain medications, which did not constitute a treatment modification (as per the study definition). Because of the nature of information in the database, details regarding the inpatient treatment could not be collected.

Medication adherence is a modifiable factor that may contribute to increased rates of hospitalization and readmission in HF patients. ${ }^{27}$ The cut-off for an adherence rate associated with significant improvement in survival is $88 \%$ in HF patients. ${ }^{28}$ Adherence estimates in the literature vary considerably, ranging from $40 \%$ to $94 \%$; this variability is attributed to the method of adherence measurement and the population under consideration. The PDC in this study ranged from 0.65 to 0.87 , suggesting that patients had moderate to good adherence. It should be noted that claims data reflect prescription fills for medications (regimens); therefore, the data presented would be impacted by patient nonadherence in filling and refilling their prescriptions. 


\section{Limitations}

Several limitations should be acknowledged when interpreting the results from this study. ICD-9-CM codes were used to identify HF diagnosis-they might not mirror confirmed clinical diagnoses and lack details to evaluate severity of illness (NYHA classification was not reported in the database). Additionally, the study cohort could not be categorized by type of HF (e.g., acute, chronic, rEF, or pEF). Therefore, the lack of clinical data on HF type and NYHA class does not allow for proper interpretation of the results and may have under- or overestimated the findings. Development of an algorithm to predict the diagnosis of HF patients will be useful while conducting such database studies. Furthermore, it is not certain whether the first recorded HF diagnosis code is when HF was truly diagnosed. Changes in index treatment combination do not account for modification of doses. Duration of therapy might have been underestimated owing to dropouts from the database. For patients who experienced index hospitalization, data on treatment before hospitalization were not available; therefore, they were not considered for the calculations. As previously noted, claims data reflect fills for the medication; therefore, the data presented are an estimate of patient nonadherence to fill the medication. Patients were not categorized as medication eligible or noneligible because of lack of clinical data; therefore, the prescription fill pattern was not estimated according to eligibility. Hence, the presented data may appear different compared with other studies that assessed eligibility. Although the patient population is derived from nationally representative groups of patients, it may not be generalizable beyond the commercially and Medicare supplement insured patient groups.

Despite these limitations, our study has considerable strengths. First, the study shows the impact of hospitalization on HF-specific treatment among newly diagnosed HF patients. Second, this large, population-based study reflects the real-world clinical practice and treatment adherence of over 200,000 HF patients in the United States. Last, persistence to medication was assessed using multiple gap periods (30 days, 45 days, and 60 days), since there is no recognized standard to assess this parameter.

\section{Conclusions}

Findings of this representative claims database analysis among 235,758 HF patients suggest that more than one third of the patients do not receive HF-specific medication within 30 days following initial HF diagnosis. Despite ACCF/AHA recommendations to initiate treatment with a combination of 2 HF drug classes, only $40 \%$ of patients had a prescription fill for the same. Hospitalization did not appear to have a major impact on HF therapy-prescribing patterns. To our knowledge, this is the first study to evaluate the impact of hospitalization on HF-specific treatment among newly diagnosed patients.
Adherence and persistence were moderate across all HF therapies of interest. This analysis reveals the need for further research in order to better understand the reasons for the demonstrated delay in HF treatment initiation and limited use of guidelines-recommended combination therapies after initial diagnosis. Investigation of the effect of this delayed therapy initiation on patient outcomes is also warranted.

\section{Authors}

CÉLINE DESCHASEAUX, MSc, Novartis Pharma AG, Basel, Switzerland; MARTIN MCSHARRY, BSc, and EIBHLIN HUDSON, BA, MA, PhD, Novartis Ireland Ltd, Dublin, Ireland; RUMJHUM AGRAWAL, MPharm, Novartis Healthcare Private Limited, Hyderabad, India; and STUART J. TURNER, BPharm, MPH, Novartis Pharmaceuticals, East Hanover, New Jersey, United States.

AUTHOR CORRESPONDENCE: Céline Deschaseaux, MSc, Novartis Pharma AG, Global Patient Access, Postfach, Basel-4002, Switzerland. E-mail: celine.deschaseaux@novartis.com.

\section{DISCLOSURES}

This study was funded by Novartis Pharma AG, Basel, Switzerland Deschaseaux, McSharry, Hudson, Agrawal, and Turner are permanent employees of Novartis.

Concept and study design were contributed by Deschaseaux, Hudson, and Turner, along with McSharry. McSharry took the lead in data collection, along with Deschaseaux, Hudson, and Turner. Data interpretation was performed by Hudson, along with the other authors. The manuscript was written by Agrawal, along with Deschaseaux and Turner, and revised by Deschaseaux and Turner, along with the other authors.

\section{REFERENCES}

1. Mozaffarian D, Benjamin EJ, Go AS, et al. Heart disease and stroke statistics-2015 update: a report from the American Heart Association. Circulation. 2015;131(4):e29-e322. Available at: http://circ.ahajournals.org/content/131/4/ e29.short. Accessed February 29, 2016.

2. Heidenreich PA, Albert NM, Allen LA, et al. Forecasting the impact of heart failure in the United States: a policy statement from the American Heart Association. Circ Heart Fail. 2013;6(3):606-19. Available at: http:// www.ncbi.nlm.nih.gov/pmc/articles/PMC3908895/pdf/nihms539387.pdf. Accessed February 15, 2016.

3. Yancy CW, Jessup M, Bozkurt B, et al. 2013 ACCF/AHA guideline for the management of heart failure: a report of the American College of Cardiology Foundation/American Heart Association Task Force on Practice Guidelines. J Am Coll Cardiol. 2013;62(16):e147-239. Available at: http://ac.els-cdn.com/ S0735109713021141/1-s2.0-S0735109713021141-main.pdf? tid=34fbfo665212-11e5-9492-00000aacb35f\&acdnat=1441267565_54d9673f03e5365759 7adcbd543c1982. Accessed February 15, 2016.

4. McMurray JJ, Adamopoulos S, Anker SD, et al. ESC Guidelines for the diagnosis and treatment of acute and chronic heart failure 2012: The Task Force for the Diagnosis and Treatment of Acute and Chronic Heart Failure 2012 of the European Society of Cardiology. Developed in collaboration with the Heart Failure Association (HFA) of the ESC. Eur Heart J. 2012;33(14):1787-847. Available at: http://eurheartj.oxfordjournals.org/content/ehj/33/14/1787.full.pdf. Accessed February 15, 2016. 
5. Roger VL, Weston SA, Redfield MM, et al. Trends in heart failure incidence and survival in a community-based population. JAMA. 2004; 292(3):344-50. Available at: http://jama.jamanetwork.com/article. aspx?articleid=199119. Accessed February 15, 2016.

6. Cowie MR, Anker SD, Cleland JGF, et al. Improving care for patients with acute heart failure before, during and after hospitalization. ESC Heart Failure. 2014;1(2):110-45. Available at: http://onlinelibrary.wiley.com/doi/10.1002/ ehf2.12021/pdf. Accessed February 15, 2016.

7. Desai AS, Stevenson LW. Rehospitalization for heart failure: predict or prevent? Circulation. 2012;126(4):501-06. Available at: http://circ.ahajournals.org/content/126/4/501.full.pdf+html. Accessed February 15, 2016.

8. Korves C, Eldar-Lissai A, Mchale J, Lafeuille MH, Hwa Ong S, Sheng Duh M. Resource utilization and costs following hospitalization of patients with chronic heart failure in the U.S. J Med Econ. 2012;15(5):925-37. Available at: http://www.tandfonline.com/doi/pdf/10.3111/13696998.2012.6 85136. Accessed February 15, 2016.

9. Blecker S, Paul M, Taksler G, Ogedegbe G, Katz S. Heart failure-associated hospitalizations in the United States. J Am Coll Cardiol. 2013;61(12):1259-67. Available at: http://www.ncbi.nlm.nih.gov/pmc/articles/PMC3838728/pdf/ nihms437980.pdf. Accessed February 15, 2016.

10. Chen J, Normand SL, Wang Y, Krumholz HM. National and regional trends in heart failure hospitalization and mortality rates for Medicare beneficiaries, 1998-2008. JAMA. 2011;306(15):1669-78. Available at: http:// www.ncbi.nlm.nih.gov/pmc/articles/PMC3688069/pdf/nihms464765.pdf. Accessed February 15, 2016.

11. Atwater BD, Dai D, Allen-Lapointe NM, et al. Is heart failure guideline adherence being underestimated? The impact of therapeutic contraindications. Am Heart J. 2012;164(5):750-55. Available at: http://ac.els-cdn.com/ S0002870312005303/1-s2.0-S0002870312005303-main.pdf?_tid=74987d7a$5217-11$ e5-8db4-00000aacb35f\&acdnat $=1441269819 \_55117$ eb54950cc235d 8de3le45b6da9a. Accessed February 15, 2016.

12. Calvin JE, Shanbhag S, Avery E, Kane J, Richardson D, Powell L. Adherence to evidence-based guidelines for heart failure in physicians and their patients: lessons from the Heart Failure Adherence Retention Trial (HART). Congest Heart Fail. 2012;18(2):73-78. Available at: http://onlinelibrary.wiley.com/doi/10.1111/j.1751-7133.2011.00263.x/pdf. Accessed February 15, 2016

13. Steinman MA, Dimaano L, Peterson CA, et al. Reasons for not prescribing guideline-recommended medications to adults with heart failure. Med Care. 2013;51(10):901-07. Available at: http://www.ncbi.nlm.nih.gov/pmc/ articles/PMC3806480/pdf/nihms-513842.pdf. Accessed February 15, 2016.

14. Skrepnek GH, Abarca J, Malone DC, Armstrong EP, Shirazi FM, Woosley RL. Incremental effects of concurrent pharmacotherapeutic regimens for heart failure on hospitalizations and costs. Annals Pharmacother. 2005;39(11):1785-91.

15. Danielson E. Health research data for the real world: the MarketScan Databases. White Paper. Truven Health Analytics. January 2014.

16. Peterson AM, Nau DP, Cramer JA, Benner J, Gwadry-Sridhar F, Nichol M. A checklist for medication compliance and persistence studies using retrospective databases. Value Health. 2007;10(1):3-12.

17. Dunlay SM, Eveleth JM, Shah ND, McNallan SM, Roger VL. Medication adherence among community-dwelling patients with heart failure. Mayo Clin Proc. 2011;86(4):273-81. Available at: http://www.ncbi.nlm.nih.gov/pmc/ articles/PMC3068886/pdf/mayoclinproc_86_4_003.pdf. Accessed February 15, 2016.
18. Korves C, Eldar-Lissai A, McHale J, Lafeuille MH, Hwa Ong S, Sheng Duh M. Resource utilization and costs following hospitalization of patients with chronic heart failure in the U.S. J Med Econ. 2012;15(5):925-37. Available at: http://www.tandfonline.com/doi/pdf/10.3111/13696998.2012.6 85136. Accessed February 15, 2016.

19. Shafazand S, Yang Y, Amore E, O’Neal W, Brixner D. A retrospective, observational cohort analysis of a nationwide database to compare heart failure prescriptions and related health care utilization before and after publication of updated treatment guidelines in the United States. Clin Ther. 2010;32(9):1642-50. Available at: http://ac.els-cdn.com/ S0149291810002626/1-s2.0-S0149291810002626-main.pdf?_tid=608dd33c5219-1le5-9998-00000aab0f27\&acdnat=1441270645_9b384cb81f7bf63b03 040545elcbfblc. Accessed February 29, 2016.

20. Mosalpuria K, Agarwal SK, Yaemsiri S, et al. Outpatient management of heart failure in the United States, 2006-2008. Tex Heart Inst J. 2014:41(3):253-61. Available at: http://www.ncbi.nlm.nih.gov/pmc/articles/ PMC4060338/pdf/i0730-2347-41-3-253.pdf. Accessed February 15, 2016.

21. Krantz MJ, Ambardekar AV, Kaltenbach L, et al. Patterns and predictors of evidence-based medication continuation among hospitalized heart failure patients (from Get With the Guidelines-Heart Failure). Am J Cardiol. 2011;107(12):1818-23. Available at: http://ac.els-cdn.com/ S0002914911010101/1-s2.0-S0002914911010101-main.pdf?_tid=c68bella5219-1le5-bacb-00000aabOf01\&acdnat=1441270816_4f1369b00f24120593 5e9f8c08178f2a. Accessed February 15, 2016.

22. Fonarow GC, Yancy CW, Albert NM, et al. Heart failure care in the outpatient cardiology practice setting: findings from IMPROVE HF. Circ Heart Fail. 2008;1(2):98-106. Available at: http://circheartfailure.ahajournals.org/ content/1/2/98.full.pdf+html. Accessed February 15, 2016.

23. Ambrosy AP, Gheorghiade M, Chioncel O, Mentz RJ, Butler J. Global perspectives in hospitalized heart failure: regional and ethnic variation in patient characteristics, management, and outcomes. Curr Heart Fail Rep. 2014;11(4):416-27.

24. Nichols GA, Reynolds K, Butler MG, Kimes TM, Chan WW. Association of acute heart failure length of stay with subsequent re-admission $\&$ allcause mortality among patients with preserved vs. reduced ejection fraction. Circulation. 2014;130:A11307. [Abstract]. Available at: http://circ.ahajournals. org/content/130/Suppl_2/A11307.abstract. Accessed February 15, 2016.

25. Fonarow GC, Abraham WT, Albert NM, et al. Age- and gender-related differences in quality of care and outcomes of patients hospitalized with heart failure (from OPTIMIZE-HF). Am J Cardiol. 2009;104(1):107-15. Available at: http://ac.els-cdn.com/S000291490900678X/1-s2.0S000291490900678X-main.pdf?_tid=c2f6223c-52ld-1le5-b8fe-00000aac b35d\&acdnat $=1441272528 \_474 b f e d f 85 e c 3 f 58 e f e 5 f 2 c 9542 f 1 b 37$. Accessed February 15, 2016.

26. Wang G, Zhang Z, Ayala C, Wall HK, Fang J. Costs of heart failurerelated hospitalizations in patients aged 18 to 64 years. Am J Manag Care. 2010;16(10):769-76

27. Riles EM, Jain AV, Fendrick AM. Medication adherence and heart failure. Curr Cardiol Rep. 2014;16(3):458

28. Wu JR, Moser DK, De Jong MJ, et al. Defining an evidence-based cutpoint for medication adherence in heart failure. Am Heart J. 2009; 157(2):285-91. Available at: http://www.ncbi.nlm.nih.gov/pmc/articles/ PMC2668834/pdf/nihms95199.pdf. Accessed February 15, 2016. 